\title{
Fourier Transform of the Continuous Arithmetic Asian Options PDE
}

\section{Zieneb Ali Elshegmani and Rokiah Rozita Ahmad}

School of Mathematical Sciences, Faculty of Science and Technology, Universiti Kebangsaan Malaysia, Bangi, 43600 Selangor, Malaysia

Correspondence should be addressed to Zieneb Ali Elshegmani, zelsheqmani@yahoo.com

Received 23 June 2011; Accepted 2 August 2011

Academic Editor: A. Bellouquid

Copyright (C) 2011 Z. A. Elshegmani and R. R. Ahmad. This is an open access article distributed under the Creative Commons Attribution License, which permits unrestricted use, distribution, and reproduction in any medium, provided the original work is properly cited.

Price of the arithmetic Asian options is not known in a closed-form solution, since arithmetic Asian option PDE is a degenerate partial differential equation in three dimensions. In this work we provide a new method for computing the continuous arithmetic Asian option price by means of partial differential equations. Using Fourier transform and changing some variables of the PDE we get a new direct method for solving the governing PDE without reducing the dimensionality of the PDE as most authors have done. We transform the second-order PDE with nonconstant coefficients to the first order with constant coefficients, which can be solved analytically.

\section{Introduction}

An Asian option is one whose payoff includes a time average of the underlying asset price. Asian options can be classified by their method of averaging, such as arithmetic or geometric. The geometric average Asian option is easy to price because a closed-form solution is available [1]. So in this work we only focus on arithmetic Asian option, which is the most commonly used, though an exact analytical solution for arithmetic average rate Asian options has not existed. This missing solution is primarily because the arithmetic average of a set of lognormal random variables is not lognormally distributed. There are several approaches to pricing arithmetic Asian options. The first approach is deriving approximations of closedform solutions. Turnbull and Wakeman [2] and Levy [3] find approximate valuation formula by matching the first several moments of the arithmetic average. Geman and Yor [4] derive an analytical solution for pricing arithmetic Asian option in terms of the inverse Laplace transform. However, this transform is only applicable in some cases. A one-dimensional PDE is derived to price Asian options contingent on dividend paying stocks by Večeř [5]. 
Vecer and $\mathrm{Xu}[6]$ show that the price of arithmetic Asian option satisfies an integrodifferential equation in the case that the underlying asset is driven by special martingale processes, of which the Levy process is a special case. Dewynne and Shaw [7] provide a simplified means of pricing arithmetic Asian options by PDE approach, they derive an analytical formula for the Laplace transform in time of the Asian option, and then they obtained asymptotic solutions for Black-Scholes PDE for Asian options for low-volatility limit which is the big problem on using Laplace transform. Cruz-Báez and González-Rodrígues [8] obtain the same solution of Geman and Yor for arithmetic Asian options using partial differential equations, integral transforms, and mathematica programming, instead Bessel's processes. Elshegmani et al. [9] derive a modified arithmetic Asian options PDE, together with its analytical solution. In addition, there are several numerical approaches to pricing arithmetic Asian options such as Monte Carlo's simulation, Binomial Tree, and Finite Element Method.

In this paper, we derive the PDE for continuous arithmetic Asian option, and give a new method for solving this equation using Fourier transform.

\section{Derivation of The PDE for Continuous Arithmetic Asian Option}

We begin by assuming that the spot price $S_{t}$ of the underlying asset of the Asian option satisfies the stochastic differential equation

$$
d S_{t}=\mu S_{t} d t+\sigma S_{t} d W_{t}
$$

where $W_{t}$ is a standard Brownian motion, $\mu$ and $\sigma$ are constants.

We now consider continuous arithmetic Asian option with the average rate defined by the running sum of the underlying asset price

$$
A_{t}=\int_{0}^{t} S_{u} d u
$$

or in differential form

$$
d A_{t}=S_{t} d t
$$

Suppose an Asian option has pay-off function $\varphi\left(S_{T}, A_{T} / T\right)$ at an expiration date $T$. Then, the value of the Arithmetic Asian option at time $t$ is

$$
V\left(t, S_{t}, A_{t}\right)=e^{-r(T-t)} E\left(\varphi\left(S_{T}, \frac{A_{T}}{T} \mid F_{t}\right)\right)
$$

By multidimension of Ito's Lemma, we have

$$
d V=\left(\frac{\partial V}{\partial t}+\mu S \frac{\partial V}{\partial S}+\frac{\sigma^{2} S^{2}}{2} \frac{\partial^{2} V}{\partial S^{2}}+S \frac{\partial V}{\partial A}\right) d t+\left(\sigma S \frac{\partial V}{\partial S}\right) d W
$$


Set up a portfolio of one Asian option and a number $\Delta$ of the underlying assets, the value of this portfolio is

$$
\Pi=V\left(t, S_{t}, A_{t}\right)-\Delta S
$$

and the change in the value of this portfolio is

$$
\begin{gathered}
d \Pi=d V\left(t, S_{t}, A_{t}\right)-\Delta d S \\
d \Pi=\left(\frac{\partial V}{\partial t}+\frac{\sigma^{2} S^{2}}{2} \frac{\partial^{2} V}{\partial S^{2}}+S \frac{\partial V}{\partial A}\right) d t+\mu S\left(\frac{\partial V}{\partial S}-\Delta\right) d S
\end{gathered}
$$

To get rid of the stochastic term, choose $\Delta=\partial V / \partial S$,

$$
d \Pi=\left(\frac{\partial V}{\partial t}+\frac{\sigma^{2} S^{2}}{2} \frac{\partial^{2} V}{\partial S^{2}}+S \frac{\partial V}{\partial A}\right) d t
$$

But we know that the change in the value of a portfolio with risk-less asset is

$$
d \Pi=r \Pi d t=r V-r S \frac{\partial V}{\partial S}
$$

From (2.9) and (2.8), yield its

$$
\begin{gathered}
\frac{\partial V}{\partial t}+\frac{1}{2} \sigma^{2} S^{2} \frac{\partial^{2} V}{\partial S^{2}}+r S \frac{\partial V}{\partial S}+S \frac{\partial V}{\partial A}-r V=0 \\
V\left(T, S_{T}, A_{T}\right)=\varphi\left(S_{T}, \frac{A_{T}}{T}\right)
\end{gathered}
$$

This is the Black-Scholes PDE of the continuous arithmetic Asian options.

There are four different types of the continuous arithmetic Asian options depending on the pay-off function as follows.

(1) Arithmetic average fixed strike call option:

$$
\varphi\left(S_{T}, \frac{A_{T}}{T}\right)=\max \left(\frac{A_{T}}{T}-k, 0\right)
$$

(2) Arithmetic average fixed strike put option:

$$
\varphi\left(S_{T}, \frac{A_{T}}{T}\right)=\max \left(k-\frac{A_{T}}{T}, 0\right)
$$


(3) Arithmetic average floating strike call option:

$$
\varphi\left(S_{T}, \frac{A_{T}}{T}\right)=\max \left(S_{T}-\frac{A_{T}}{T}, 0\right) .
$$

(4) Arithmetic average floating strike put option:

$$
\varphi\left(S_{T}, \frac{A_{T}}{T}\right)=\max \left(\frac{A_{T}}{T}-S_{T}, 0\right) .
$$

\section{Analytical Solution of The PDE}

To solve (2.10) we will make the first change of the variables

$$
\begin{gathered}
V(t, S, A)=e^{r t} f(t, S, A), \\
\frac{\partial f}{\partial t}+\frac{1}{2} \sigma^{2} S^{2} \frac{\partial^{2} f}{\partial S^{2}}+r S \frac{\partial f}{\partial S}+S \frac{\partial f}{\partial A}=0 .
\end{gathered}
$$

Assume further $z=\ln s, s>0, \tau=T-t$, and taking into account that

$$
S^{2} \frac{\partial^{2} V}{\partial S^{2}}=\frac{\partial^{2} f}{\partial z^{2}}-\frac{\partial f}{\partial z}, S \frac{\partial f}{\partial S}=\frac{\partial f}{\partial z}
$$

then (3.1) becomes:

$$
\begin{aligned}
& -\frac{\partial f}{\partial \tau}+\frac{\sigma^{2}}{2}\left(\frac{\partial^{2} f}{\partial z^{2}}-\frac{\partial f}{\partial z}\right)+r \frac{\partial f}{\partial z}+e^{z} \frac{\partial f}{\partial A}=0 \\
& -\frac{\partial f}{\partial \tau}+\frac{\sigma^{2}}{2} \frac{\partial^{2} f}{\partial z^{2}}+\left(r-\frac{\sigma^{2}}{2}\right) \frac{\partial f}{\partial z}+e^{z} \frac{\partial f}{\partial A}=0 .
\end{aligned}
$$

Assume $z=-i y$ where $i$ is a complex number, then we get

$$
-\frac{\partial f}{\partial \tau}-\frac{\sigma^{2}}{2} \frac{\partial^{2} f}{\partial y^{2}}-i\left(r-\frac{\sigma^{2}}{2}\right) \frac{\partial f}{\partial y}+e^{-i y} \frac{\partial f}{\partial A}=0 .
$$

Note that all the coefficients in the above equation are constant except the coefficient with the term $\partial f / \partial A$, so we can easily apply the Fourier transform.

Fourier's transform for a function $f(x)$ is defined by

$$
F\{f(x)\}=g(\omega)=\int_{-\infty}^{\infty} f(x) e^{-i \omega x} d x
$$


and the inverse Fourier transform is

$$
F^{-1}\{g(\omega)\}=f(x)=\frac{1}{2 \pi} \int_{-\infty}^{\infty} g(\omega) e^{i \omega x} d \omega
$$

Some properties of the Fourier transform that we need in this work as follows:

$$
\begin{gathered}
F\left\{\frac{\partial^{n} f}{\partial x^{n}}\right\}=(i \omega)^{n} g(\omega), \\
F\left[e^{i a x} f(x)\right]=g(\omega-a), \\
F[f(x-a)]=e^{-i a \omega} g(\omega), \\
F\left[e^{i a x}\right]=\delta(\omega-a),
\end{gathered}
$$

where $a$ is a constant, and $\delta(\omega)$ is a Dirac delta function.

Applying the Fourier transform in $z$ on (3.4),

$$
\begin{gathered}
-\frac{\partial g(\tau, \omega, A)}{\partial \tau}+\frac{\sigma^{2} \omega^{2}}{2} g(\tau, \omega, A)+\omega\left(r-\frac{\sigma^{2}}{2}\right) g(\tau, \omega, A)+\frac{\partial g(\tau, \omega+1, A)}{\partial A}=0 \\
\frac{\partial g(\tau, \omega, A)}{\partial \tau}=\left[\frac{\sigma^{2} \omega^{2}}{2}+\omega\left(r-\frac{\sigma^{2}}{2}\right)\right] g(\tau, \omega, A)+\frac{\partial g(\tau, \omega+1, A)}{\partial A}
\end{gathered}
$$

Assume

$$
g(\tau, \omega, A)=e^{\left[\left(\sigma^{2} \omega^{2} / 2\right)+\omega\left(r-\left(\sigma^{2} / 2\right)\right)\right] \tau} h(\tau, \omega, A) .
$$

Then (3.9) is reduced to

$$
\frac{\partial h(\tau, \omega, A)}{\partial \tau}=\frac{\partial h(\tau, \omega+1, A)}{\partial A}
$$

Applying another Fourier transform in $\omega$,

$$
\frac{\partial \widehat{h}(\tau, \widehat{\omega}, A)}{\partial \tau}=\frac{\partial \widehat{h}(\tau, \widehat{\omega}, A)}{\partial A} e^{i \widehat{\omega}} .
$$

One solution of the above equation is

$$
\widehat{h}(\tau, \widehat{\omega}, A)=c\left(\tau+A e^{-i \widehat{\omega}}\right) .
$$


From the initial condition, we have

$$
\begin{gathered}
\widehat{h}(0, \widehat{\omega}, A)=\varphi(\widehat{\omega}, A)=c A e^{-i \hat{\omega}}, \\
c=\frac{\varphi(\widehat{\omega}, A)}{A e^{-i \widehat{\omega}}}=\widehat{\varphi}(\widehat{\omega}, A), \\
\widehat{h}(\tau, \widehat{\omega}, A)=\widehat{\varphi}(\widehat{\omega}, A)\left(\tau+A e^{-i \widehat{\omega}}\right) .
\end{gathered}
$$

Applying the inverse Fourier transform in $\widehat{\omega}$,

$$
\begin{gathered}
h(\tau, \omega, A)=\varphi(\omega, A)[\tau \delta(\omega)+A \delta(\omega-1)] \\
g(\tau, \omega, A)=\varphi(\omega, A)[\tau \delta(\omega)+A \delta(\omega-1)] e^{\left[\left(\sigma^{2} \omega^{2} / 2\right)+\omega\left(r-\left(\sigma^{2} / 2\right)\right)\right] \tau} \\
f(\tau,-i z, A)=F^{-1}[g(\tau, \omega, A)] \\
f(\tau, y, A)=\frac{1}{2 \pi} \int_{-\infty}^{\infty}(\varphi(\omega, A)[\tau \delta(\omega)+A \delta(\omega-1)]) e^{\left[\left(\sigma^{2} \omega^{2} / 2\right)+\omega\left(r-\left(\sigma^{2} / 2\right)\right)\right] \tau} e^{-i \omega z} d \omega \\
f(\tau, z, A)=\frac{1}{2 \pi} \int_{-\infty}^{\infty}(\varphi(\omega, A)[\tau \delta(\omega)+A \delta(\omega-1)]) e^{\left[\left(\sigma^{2} \omega^{2} / 2\right)+\omega\left(r-\left(\sigma^{2} / 2\right)\right] \tau\right.} e^{\omega z} d \omega \\
f(t, S, A)=\frac{1}{2 \pi} \int_{-\infty}^{\infty} S^{\omega}(\varphi(\omega, A)[(T-t) \delta(\omega)+A \delta(\omega-1)]) e^{\left[\left(\sigma^{2} \omega^{2} / 2\right)+\omega\left(r-\left(\sigma^{2} / 2\right)\right)\right](T-t)} d \omega \\
V(t, S, A)=\frac{1}{2 \pi} \int_{-\infty}^{\infty} S^{\omega}(\varphi(\omega, A)[(T-t) \delta(\omega)+A \delta(\omega-1)]) e^{\left[\left(\sigma^{2} \omega^{2} / 2\right)+\omega\left(r-\left(\sigma^{2} / 2\right)\right)-r\right](T-t)} d \omega
\end{gathered}
$$

To prove that expression (3.21) is a direct solution for (2.1), we will differentiate, (3.14) with respect to all variables, and then substituting into (2.1),

$$
\begin{aligned}
\frac{\partial V}{\partial t}= & -\left[\frac{\sigma^{2} \omega^{2}}{2}+\omega\left(r-\frac{\sigma^{2}}{2}\right)-r\right] V(t, S, A) \\
& -\frac{1}{2 \pi} \varphi(\omega, A) \int_{-\infty}^{\infty} S^{\omega} \delta(\omega) e^{\left[\left(\sigma^{2} \omega^{2} / 2\right)+\omega\left(r-\left(\sigma^{2} / 2\right)\right)-r\right](T-t)} d \omega, \\
\frac{\partial V}{\partial S}= & \omega S^{\omega-1} V(t, S, A), \\
\frac{\partial^{2} V}{\partial S^{2}}= & \omega(\omega-1) S^{\omega-2} V(t, S, A), \\
\frac{\partial V}{\partial A}= & \frac{1}{2 \pi} \int_{-\infty}^{\infty} S^{i \omega}(\varphi(\omega, A)[\delta(\omega-1)]) e^{\left[\left(\sigma^{2} \omega^{2} / 2\right)+\omega\left(r-\left(\sigma^{2} / 2\right)\right)-r\right](T-t)} d \omega .
\end{aligned}
$$


Substituting (3.22) into (2.10) yields

$$
\begin{aligned}
\frac{\partial V}{\partial t}+ & \frac{\sigma^{2} S^{2}}{2} \frac{\partial^{2} V}{\partial S^{2}}+r S \frac{\partial V}{\partial S}+S \frac{\partial V}{\partial A}-r V \\
= & -\left[\frac{\sigma^{2} \omega^{2}}{2}+\omega\left(r-\frac{\sigma^{2}}{2}\right)-r\right] V+\frac{\sigma^{2} S^{2}}{2} \omega(\omega-1) S^{\omega-2} V \\
& +\frac{-1}{2 \pi} \int_{-\infty}^{\infty} \varphi(\omega, A) S^{\omega} \delta(\omega) e^{\left[\left(\sigma^{2} \omega^{2} / 2\right)+\omega\left(r-\left(\sigma^{2} / 2\right)\right)-r\right](T-t)} d \omega+r S \omega S^{\omega-1} V \\
& +S \frac{1}{2 \pi} \int_{-\infty}^{\infty} S^{\omega}(\varphi(\omega, A)[\delta(\omega-1)]) e^{\left[\left(\sigma^{2} \omega^{2} / 2\right)+\omega\left(r-\left(\sigma^{2} / 2\right)\right)-r\right](T-t)} d \omega-r V \\
= & -\frac{\sigma^{2} \omega^{2}}{2}-r \omega+\frac{\sigma^{2} \omega}{2}+r+\frac{\sigma^{2} \omega^{2}}{2}-\frac{\sigma^{2} \omega}{2}+r \omega-r \\
& +\frac{1}{2 \pi} \int_{-\infty}^{\infty} S^{\omega} \varphi(\omega, A)[S \delta(\omega-1)-\delta(\omega)] e^{\left[\left(\sigma^{2} \omega^{2} / 2\right)+\omega\left(r-\left(\sigma^{2} / 2\right)\right)-r\right](T-t)} d \omega=0
\end{aligned}
$$

since we have $S=e^{-i z}$,

$$
\begin{gathered}
S \delta(\omega-1)=e^{-i z} \delta(\omega-1)=\int_{-\infty}^{\infty} e^{-i z} e^{-i z(\omega-1)} d \omega \\
=\int_{-\infty}^{\infty} e^{-i z(\omega-1+1)}=\int_{-\infty}^{\infty} e^{-i z \omega} d \omega=\delta(\omega),
\end{gathered}
$$

so

$$
\int_{-\infty}^{\infty} S^{i \omega}[\delta(\omega)-\delta(\omega)] e^{\left[\left(\sigma^{2} \omega^{2} / 2\right)+\omega\left(r-\left(\sigma^{2} / 2\right)\right)-r\right](T-t)} d \omega=0
$$

\section{Conclusion}

The valuation of the arithmetic Asian options with continuous sampling has been an outstanding issue in finance for several decades. Describing the distribution of the integral of lognormals is found to be challenging. In this paper, we have solved the problem with the PDE approach. We show that the governing PDE from the second order can be transformed to a simple PDE from the first order with constant coefficients, which can be easily solved. We have the solution for all types of the continuous arithmetic Asian options only by changing the pay-off function with respect to which one of the options we want to price. Our approach could be extended to the continuous arithmetic Asian options with constant dividend yield. 


\section{References}

[1] E. Barucci, S. Polidoro, and V. Vespri, "Some results on partial differential equations and Asian options," Mathematical Models \& Methods in Applied Sciences, vol. 11, no. 3, pp. 475-497, 2001.

[2] S. M. Turnbull and L. M. Wakeman, "Aquick algorithm for pricing European average options," Journal of Financial and Quantitative Analysis, vol. 26, pp. 377-389, 1991.

[3] E. Levy, "Pricing European average rate currency options," Journal of International Money and Finance, vol. 11, pp. 474-491, 1992.

[4] H. Geman and M. Yor, "Bessel processes, Asian options, and perpetuities," Mathematical Finance, vol. 3, pp. 349-375, 1993.

[5] J. Vecer, "A new PDE approach for pricing arithmetic average Asian option," Journal of Computational Finance, vol. 4, pp. 105-113, 2001.

[6] J. Večeř and M. Xu, "Pricing Asian options in a semimartingale model," Quantitative Finance, vol. 4, no. 2, pp. 170-175, 2004.

[7] J. N. Dewynne and W. T. Shaw, "Differential equations and asymptotic solutions for arithmetic Asian options: 'Black-Scholes formulae' for Asian rate calls," European Journal of Applied Mathematics, vol. 19, no. 4, pp. 353-391, 2008.

[8] D. I. Cruz-Báez and J. M. González-Rodríguez, "A different approach for pricing Asian options," Journal of Applied Mathematics Letters, vol. 21, no. 3, pp. 303-306, 2008.

[9] Z. A. Elshegmani, R. R. Ahmad, and S. H. Jaaman, "On the modified arithmetic Asian option equation and its analytical solution," Applied Mathematical Sciences, vol. 5, pp. 1217-1227, 2011. 


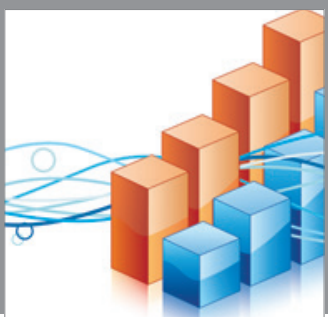

Advances in

Operations Research

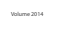

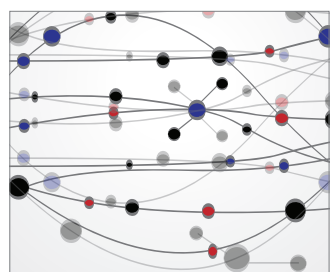

\section{The Scientific} World Journal
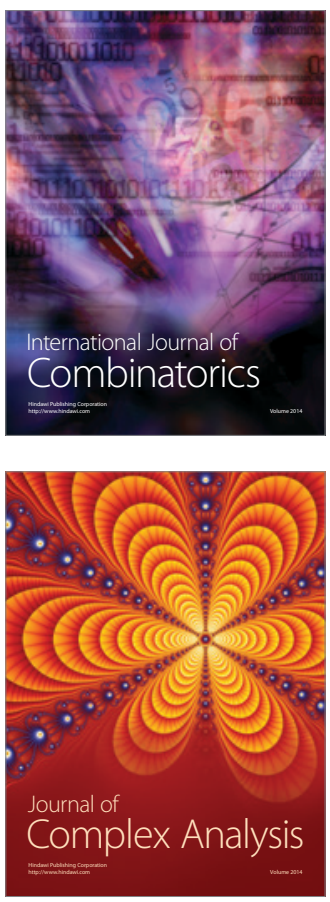

International Journal of

Mathematics and

Mathematical

Sciences
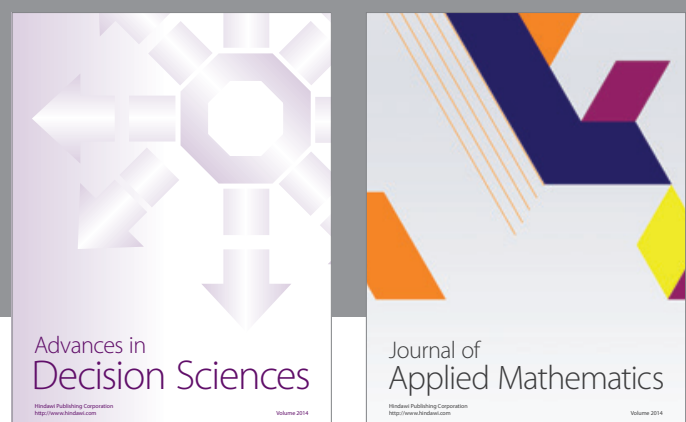

Journal of

Applied Mathematics
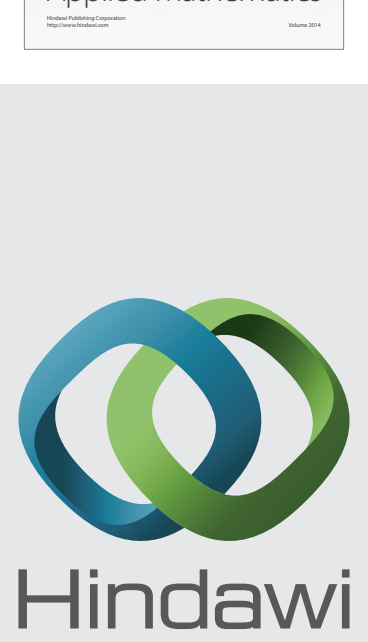

Submit your manuscripts at http://www.hindawi.com
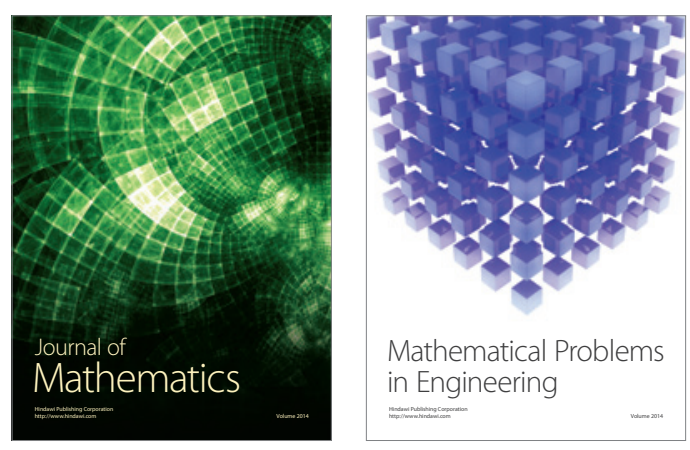

Mathematical Problems in Engineering
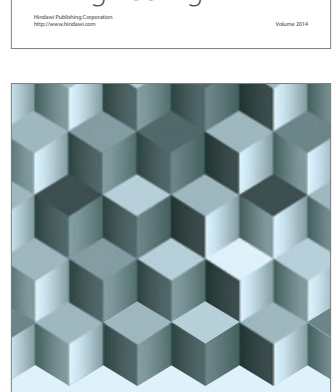

Journal of

Function Spaces
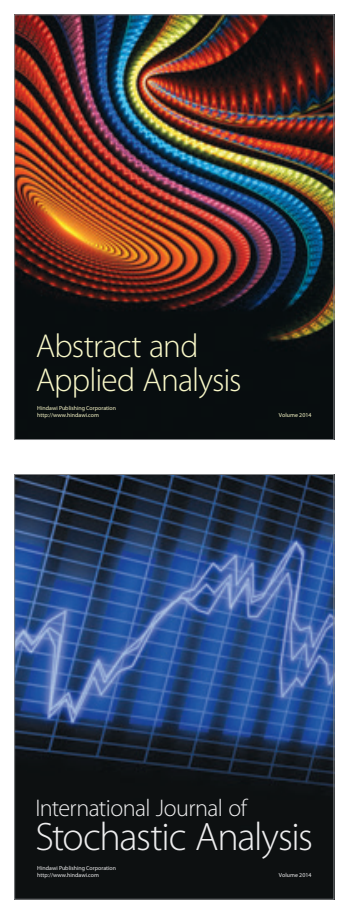

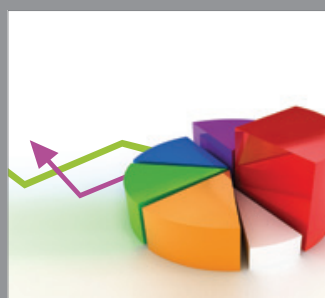

ournal of

Probability and Statistics

Promensencen
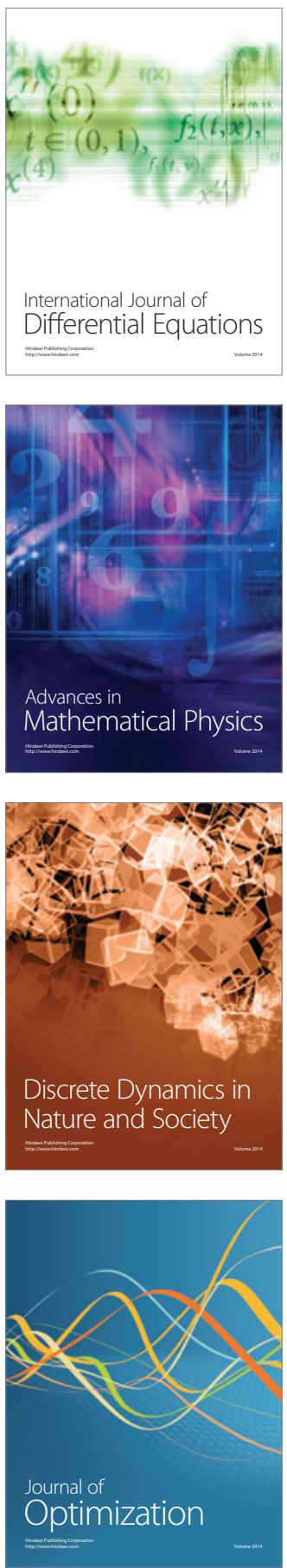